\title{
Support System for Determination of Low-Income Students Scholarship (BSM) with Technique for Order Preference by Similarity to Ideal Solution (TOPSIS)
}

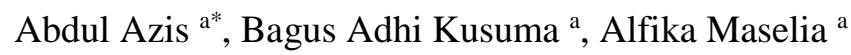 \\ ${ }^{a}$ Faculty of Computer Science, Universitas Amikom Purwokerto \\ * corresponding author
}

\begin{abstract}
Muhammadiyah 3 Middle School in Purwokerto is the school that organizes the Low-Income Students Scholarship (BSM) program every first semester held in each new school year. During this time, processing student data and other equipment have been processed with manual calculations, as well as data storage using only Microsoft Excel. In selecting ranking, it still uses paper. The paper calculation on the selection of BSM recipients in the previous year is often lost and hard to find already needed, also there is no particular system for processing the data so that the subjective method is still needed by relying trusts on personal. The purpose of this study is the creation of a Decision Support System (DSS) application for Determining Low-Income Students Scholarship (BSM) using the Technique for Order Preference by Similarity to Ideal Solution (TOPSIS) method at Muhammadiyah 3 Middle School in Purwokerto so that the selection process of Low-Income Students Scholarship (BSM) can be used. So that it is right on target to students who are entitled to BSM and can store data safely. The system development method used is a waterfall.
\end{abstract}

Keywords: Decision Support System; Technique for Order Preference by Similarity to Ideal Solution (TOPSIS); Website; Muhammadiyah 3 Middle School in Purwokerto.

\section{Introduction}

Muhammadiyah 3 Middle School in Purwokerto is one of the private schools established in 1989, based on Islamic religion and found in the city of Purwokerto with the address Jl. Dr. Number No. 79 Bancarkembar North Purwokerto. Muhammadiyah 3 Middle School in Purwokerto is conduct the Low-Income Students Scholarship Program (BSM) at the beginning of each semester are held on each new school year. Currently, student data and other fittings are processed with manual calculations, as well as data storage using only Microsoft Excel. In the attachment, the fishing still uses paper. Calculation paper on BSM receiver screening in previous years is often lost and difficult to find when it is needed, and there is no specific system to process the data so that the way of judgment is still subjective by relying on personal views.

On the system that has been running, the time required by the BSM manager is about a year from the new school year. Data security in Microsoft Excel becomes one of the vulnerable factors due to the absence of restriction of access in its use, then in case of damage to hardware will cause data loss [1]. The absence of a system makes the assessment unobjective and resulted in errors in the recommendation of determining students who are entitled to BSM, and it is necessary a system that can help the job of decision-making BSM and can store data securely and efficiently searchable by using databases. Decision Support System with the method of TOPSIS data selectors, the student candidate of the BSM receiver is carried out through the criteria and predefined weights so that it can help to determine the feasibility of receiving Low-Income Students Scholarship Program accurately.

TOPSIS uses the principle that the selected alternatives should have the closest distance from the ideal positive solution and the longest distance (farthest) of the ideal negative solution from a geometric point of view using Euclidean distance (distance between two points) to determine the relative proximity of an alternative with optimal solution [2].

The purpose of this research is to make the application of the decision-making system (DSS) for Low-Income Students Scholarship (BSM) using the Technique for Order Preference by Similarity to Ideal Solution (TOPSIS) at 
Abdu1 Azis, Bagus Adhi Kusuma, Alfika Maselia / IJIIS Vo1. 3, No. 1, March 2020, pp. 1-11

Muhammadiyah 3 Middle School in Purwokerto to facilitate the process of screening for Low-Income Students Scholarship (BSM) so that the target students who are eligible for BSM and can store data securely.

\section{Research Methods}

\subsection{System Development Methods}

Here are the stages of the system development method using waterfall [3].

a. Determination and analysis of specifications

In this stage, analysis of the needs of the software that is analyzed functional needs and analysis of non-functional needs.

b. System design and Software

The design Model used in this study uses UML (Unified Modelling Language).

c. Unit implementation and trial

In the implementation phase and unit trials, researchers in creating the system could be implemented using the Laravel Framework with its programming language PHP (PHP Hypertext Pre-processor) and MySQL used for the creation of its database.

d. System integration and trial

In this research, researchers use BlackBox testing.

e. Operation and Maintenance

The system installed and used. Maintenance includes corrected errors that were not found in the previous step. Improved implementation of system units and improved system services as new needs were found.

\subsection{Method of TOPSIS (Technique for Order Performance by Similarity to Ideal Solution)}

TOPSIS considers both the distance to the ideal solution positively and the distance to the ideal negative solution by taking the proximity relative to the ideal positive solution. Based on comparisons to their relative distances, alternative priority arrangements can be achieved. This method is widely used to complete decision-making practices. This is because the concept is understandable and straightforward, the computation is efficient, and can measure the relative performance of the alternative decisions [2].

\section{Results and Discussion}

\subsection{System Design}

Use Case Diagram 
Abdu1 Azis, Bagus Adhi Kusuma, A7fika Maselia / IJIIS Vol. 3, No. 1, March 2020, pp. 1-11

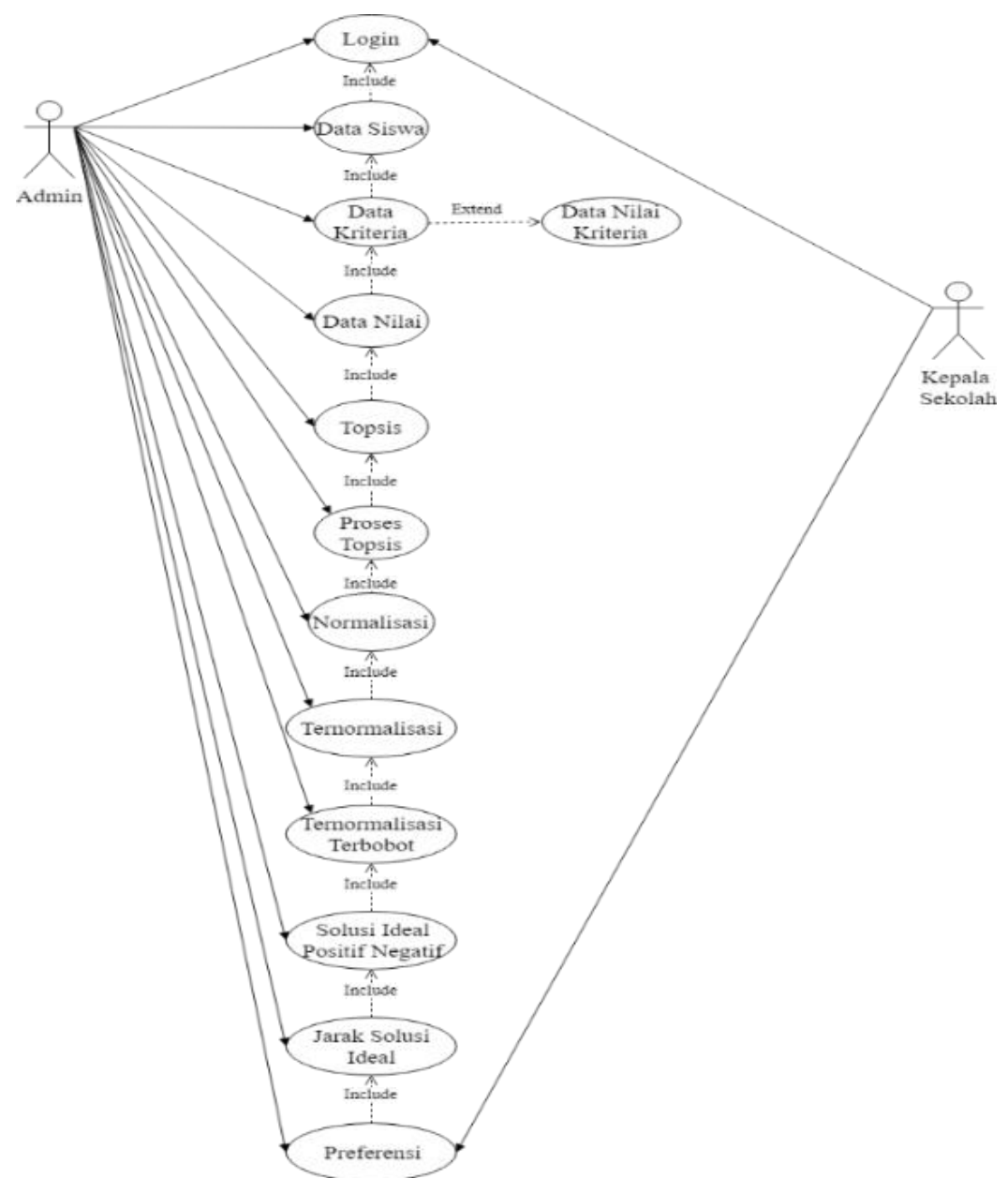

Fig. 1 Use case diagram at Muhammadiyah 3 Middle School in Purwokerto

3.2. System Results

a. Value Data Preference

In this stage, analysis of the needs of the software that is analyzed functional needs and analysis of non-functional needs.

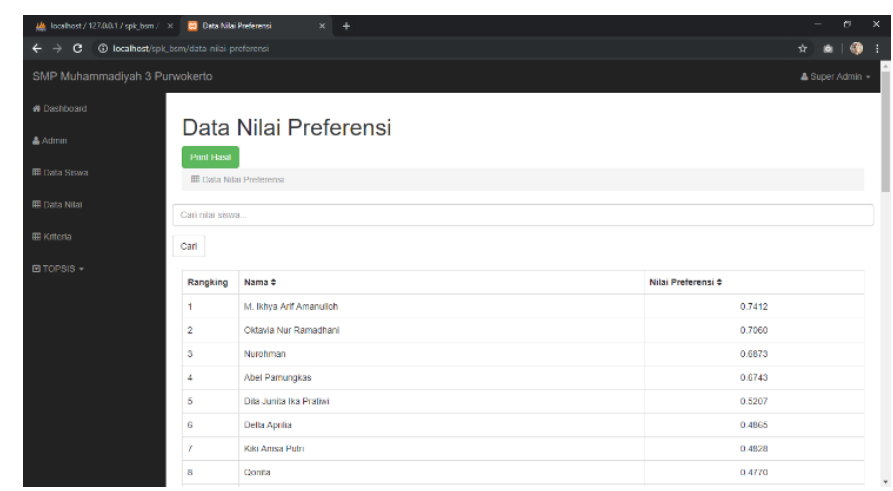

Fig. 2. Value Preference Data

Figure 2 is a preference value data that contains ranking, student names, and preference values.

b. Report 
Abdu1 Azis, Bagus Adhi Kusuma, Alfika Maselia / IJIIS Vo1. 3, No. 1, March 2020, pp. 1-11

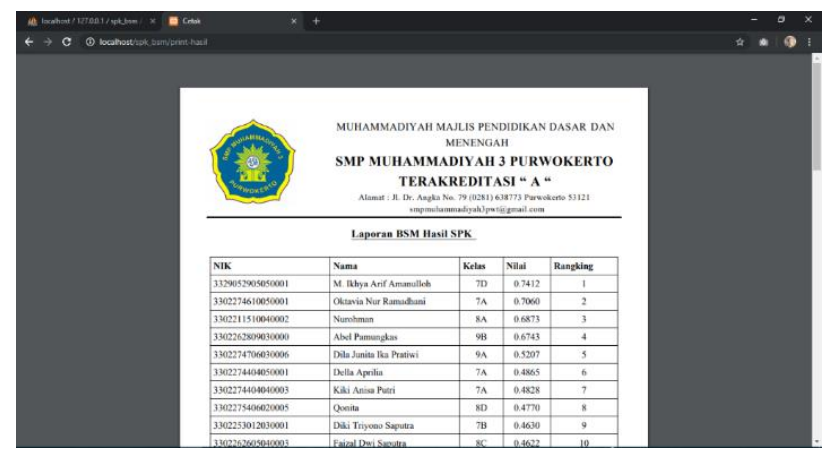

Fig. 3 Report

Figure 3.3 is the result of a report containing NIK, name, class, value, and rank.

c. Manual TOPSIS Result

1) Alternate Data

Table 1 is an alternative data taken from the students in Muhammadiyah 3 Middle School in Purwokerto, the alternative that will be used in the TOPSIS process.

Table 1. TOPSIS Alternative Data

\begin{tabular}{|l|l|l|l|}
\hline No. & Code & Alternate Names & Address \\
\hline 1. & A1 & Abel Pamungkas & Kombas \\
\hline 2. & A2 & Adika Caksana Putra Wibowo & Kutasari \\
\hline 3. & A3 & Agung Jatmiko & Arcawinangun \\
\hline 4. & A4 & Akbar Refandi & Purbalingga \\
\hline 5. & A5 & Alfi Nur Aziz & Arcawinangun \\
\hline
\end{tabular}

2) Criterion-weighted Data

Table 2 is the criteria for weighting a criterion consisting of code, criteria, weights, and properties. The contents of the table below are the data to be used in the TOPSIS process.

Table 2. TOPSIS Criterion-weighted Data

\begin{tabular}{|l|l|l|l|l|}
\hline No. & Code & Criteria & Weights & Nature \\
\hline 1. & C1 & SKTM & 0.2 & Benefit \\
\hline 2. & C 2 & PIP Recipients & 0.1 & Benefit \\
\hline 3. & C 3 & Father's income & 0.1 & Cost \\
\hline 4. & C 4 & Father's job & 0.05 & Cost \\
\hline 5. & C5 & Mother Income & 0.05 & Cost \\
\hline 6. & C6 & Mother's job & 0.05 & Cost \\
\hline 7. & C7 & KIP Recipients & 0.1 & Cost \\
\hline 8. & C 8 & KPS Recipients & 0.05 & Benefit \\
\hline 9. & C 9 & Transportation tool & 0.05 & Cost \\
\hline 10. & C10 & Type of residence & 0.05 & Cost \\
\hline 11. & C11 & Home ownership & 0.05 & Cost \\
\hline 12. & C12 & Number of Relatives & 0.025 & Benefit \\
\hline 13. & C13 & Children number ... & 0.025 & Cost \\
\hline 14. & C114 & Morals & 0.1 & Benefit \\
\hline
\end{tabular}

3) Criterion-weighted Data

Table 3 is value data per criteria such as SKTM, PIP recipient, father income, father's job, mother's income, mother's job, KIP recipient, KPS receiver, transportation tool, type of residence, homeownership, number of relatives, children number, and morals. The value in that criterion will be the value of each alternative in the TOPSIS process. 
Abdul Azis, Bagus Adhi Kusuma, A7fika Maselia / IJIIS Vol. 3, No. 1, March 2020, pp. 1-11

Table 3. Value of TOPSIS Criterion Data

\begin{tabular}{|c|c|c|c|}
\hline No. & Criteria & Description & Value \\
\hline \multirow[t]{2}{*}{1.} & \multirow[t]{2}{*}{ SKTM } & Yes & 9 \\
\hline & & Not & 1 \\
\hline \multirow[t]{2}{*}{2.} & \multirow[t]{2}{*}{ PIP Recipients } & Yes & 9 \\
\hline & & Not & 1 \\
\hline \multirow[t]{5}{*}{3.} & \multirow[t]{3}{*}{ Father's income } & No income & 9 \\
\hline & & $<500,000$ & 7 \\
\hline & & $500,000-999,999$ & 5 \\
\hline & & 1 million - 1,999,999 & 3 \\
\hline & & 2 million - 4,999,999 & 1 \\
\hline \multirow[t]{9}{*}{4.} & \multirow[t]{9}{*}{ Father's job } & Deceased & 9 \\
\hline & & Labor & 8 \\
\hline & & Farmers & 7 \\
\hline & & Small traders & 6 \\
\hline & & Other & 5 \\
\hline & & Private employees & 4 \\
\hline & & Self-employed & 3 \\
\hline & & Retired & 2 \\
\hline & & Civil Servant/Police/Military & 1 \\
\hline \multirow[t]{5}{*}{5.} & \multirow[t]{5}{*}{ Mother Income } & No income & 9 \\
\hline & & $<500,000$ & 7 \\
\hline & & 500,000 S. D 999,999 & 5 \\
\hline & & 1 million s. D 1,999,999 & 3 \\
\hline & & 2 million S. D 4,999,999 & 1 \\
\hline \multirow[t]{9}{*}{6.} & \multirow[t]{9}{*}{ Mother's job } & Deceased & 9 \\
\hline & & Not working & 8 \\
\hline & & Labor & 7 \\
\hline & & Farmers & 6 \\
\hline & & Small traders & 5 \\
\hline & & Other & 4 \\
\hline & & Private employees & 3 \\
\hline & & Self-employed & 2 \\
\hline & & Civil Servant/Police/Military & 1 \\
\hline \multirow[t]{2}{*}{7.} & \multirow[t]{2}{*}{ KIP Recipients } & Yes & 1 \\
\hline & & Not & 9 \\
\hline \multirow[t]{2}{*}{8.} & \multirow[t]{2}{*}{ KPS Recipients } & Yes & 9 \\
\hline & & Not & 1 \\
\hline \multirow[t]{5}{*}{9.} & \multirow[t]{5}{*}{ Transportation equipment } & Public transportation & 9 \\
\hline & & Private vehicle & 7 \\
\hline & & Shuttle Service & 5 \\
\hline & & Bicycle & 3 \\
\hline & & Walk & 1 \\
\hline \multirow[t]{3}{*}{10.} & \multirow[t]{3}{*}{ Type of stay } & Parlors & 9 \\
\hline & & Guardian & 6 \\
\hline & & Parents & 3 \\
\hline \multirow[t]{5}{*}{11.} & \multirow[t]{5}{*}{ Homeownership } & Do not have & 9 \\
\hline & & Dormitory & 7 \\
\hline & & Contract/Lease & 5 \\
\hline & & Private & 3 \\
\hline & & Service & 1 \\
\hline
\end{tabular}


Abdu1 Azis, Bagus Adhi Kusuma, Alfika Maselia / IJIIS Vo1. 3, No. 1, March 2020, pp. 1-11

\begin{tabular}{|l|l|l|l|}
\hline No. & Criteria & Description & Value \\
\hline \multirow{2}{*}{12.} & \multirow{2}{*}{ Number of Relatives } & $>5$ & 9 \\
\cline { 3 - 4 } & & 4 & 7 \\
\cline { 3 - 4 } & & 3 & 5 \\
\cline { 3 - 4 } & & 2 & 3 \\
\cline { 3 - 4 } & & 1 & 1 \\
\hline 13. & Children to & 1 & 9 \\
\cline { 3 - 4 } & & 2 & 7 \\
\cline { 3 - 4 } & & 3 & 3 \\
\cline { 3 - 4 } & & 4 & 1 \\
\hline 14. & Morals & 5 & 9 \\
\hline
\end{tabular}

4) Decision Matrix

Table 4 the decision matrix is a value on every alternative/student obtained from the value data on each criterion. The alternative value below will be used for the TOPSIS process.

Table 4. TOPSIS Decision Matrix

\begin{tabular}{|l|l|l|l|l|l|l|l|l|l|l|l|l|l|l|l|}
\hline No. & Name & C1 & C2 & C3 & C4 & C5 & C6 & C7 & C8 & C9 & C10 & C11 & C12 & C13 & C14 \\
\hline 1. & A1 & 9 & 9 & 9 & 9 & 5 & 7 & 1 & 1 & 1 & 3 & 3 & 5 & 4 & 9 \\
\hline 2. & A2 & 1 & 1 & 1 & 3 & 9 & 9 & 1 & 1 & 9 & 6 & 3 & 3 & 6 & 1 \\
\hline 3. & A3 & 9 & 1 & 5 & 6 & 5 & 5 & 1 & 9 & 1 & 3 & 3 & 3 & 8 & 1 \\
\hline 4. & A4 & 9 & 1 & 7 & 8 & 7 & 7 & 1 & 1 & 1 & 9 & 9 & 3 & 6 & 9 \\
\hline 5. & A5 & 1 & 1 & 5 & 8 & 9 & 8 & 1 & 1 & 1 & 3 & 3 & 5 & 4 & 1 \\
\hline
\end{tabular}

The following will explain the completion stage in the manual calculation process of the decision support system using the Technique for Order Performance by Similarity to Ideal Solution (TOPSIS) method.

a. Calculating the normalized decision matrix

$$
r_{i j}=\frac{x_{i j}}{\sqrt{\sum_{i=1}^{m} x_{i j}^{2}}}
$$

\section{Formula TOPSIS Normalization}

Description:

$r_{i j}$ : An $R$ normalized decision matrix element

$x_{i j}$ : Decision matrix Element $\mathrm{X}$

I : Alternative to $1.2, \ldots . . I$

$J$ : Criteria to $1.2, \ldots . . J$

b. Create a normalized matrix example:

$$
\begin{aligned}
& \left|\mathrm{X}_{1}\right|==15.6524 \sqrt{9^{2}+1^{2}+9^{2}+9^{2}+1^{2}} \\
& \mathrm{R}_{11}==0.5749 \frac{X 11}{|X i|} \frac{9}{15,6524} \\
& \mathrm{R}_{21}==0.0638 \frac{X 21}{|X i|} \frac{1}{15,6524} \\
& \mathrm{R}_{31}==0.5749 \frac{X 31}{|X i|} \frac{9}{15,6524}
\end{aligned}
$$


Abdul Azis, Bagus Adhi Kusuma, Alfika Maselia / IJIIS Vol. 3, No. 1, March 2020, pp. 1-11

$$
\begin{aligned}
& \mathrm{R}_{41}==0.5749 \frac{X 41}{|X i|} \frac{9}{15,6524} \\
& \mathrm{R}_{51}==0.063 \frac{X 51}{|X i|} \frac{1}{15,6524} 8
\end{aligned}
$$

Table 5 is the result of the normalized matrix table results that are in the can of the student grades of the decision matrix in the normalized TOPSIS process.

Table 5. Normalized Decision Matrix

\begin{tabular}{|l|l|l|l|l|l|l|l|l|l|l|l|l|l|}
\hline 0.5749 & 0.9761 & 0.6689 & 0.5647 & 0.3094 & 0.4275 & 0.4472 & 0.1084 & 0.1084 & 0.25 & 0.2773 & 0.5698 & 0.3086 & 0.7006 \\
\hline 0.0638 & 0.1084 & 0.0743 & 0.1882 & 0.5570 & 0.5497 & 0.4472 & 0.1084 & 0.9761 & 0.5 & 0.2773 & 0.3418 & 0.4629 & 0.0778 \\
\hline 0.5749 & 0.1084 & 0.3716 & 0.3764 & 0.3094 & 0.3054 & 0.4472 & 0.9761 & 0.1084 & 0.25 & 0.2773 & 0.3418 & 0.6172 & 0.0778 \\
\hline 0.5749 & 0.1084 & 0.5203 & 0.5019 & 0.4332 & 0.4275 & 0.4472 & 0.1084 & 0.1084 & 0.75 & 0.8320 & 0.3418 & 0.4629 & 0.7006 \\
\hline 0.0638 & 0.1084 & 0.3716 & 0.5019 & 0.5570 & 0.4886 & 0.4472 & 0.1084 & 0.1084 & 0.25 & 0.2773 & 0.5698 & 0.3086 & 0.0778 \\
\hline
\end{tabular}

c. Calculate weighted normalized decision matrix

The normalized decision matrix is weighted, the decision matrix is normalized in step 1 multiplied by the weights of each criterion i.e. $0.2,0.1,0.1,0.05,0.05,0.05,0.1,0.05,0.05,0.05,0.05,0.025,0.025,0.1$. The following table 6 is the result of the weight multiplied by the weighted normalized result.

$$
y_{i j}=w_{j} . r_{i j}
$$

Table 6. Normalized Decision Matrix

\begin{tabular}{|c|c|c|c|c|c|c|c|c|c|c|c|c|c|}
\hline 0,2 & 0,1 & 0,1 & 0,05 & 0,05 & 0,05 & 0,1 & 0,05 & 0,05 & 0,05 & 0,05 & 0,025 & 0,025 & 0,1 \\
\hline 0.1149 & 0.0976 & 0.0668 & 0.0282 & 0.0154 & 0.0213 & 0.0447 & 0.0054 & 0.0054 & 0.0125 & 0.0138 & 0.0142 & 0.0077 & 0.0700 \\
\hline 0.0127 & 0.0108 & 0.0074 & 0.0094 & 0.0278 & 0.0274 & 0.0447 & 0.0054 & 0.0488 & 0.025 & 0.0138 & 0.0085 & 0.0115 & 0.0077 \\
\hline 0.1149 & 0.0108 & 0.0371 & 0.0188 & 0.0154 & 0.0152 & 0.0447 & 0.0488 & 0.0054 & 0.0125 & 0.0138 & 0.0085 & 0.0154 & 0.0077 \\
\hline 0.1149 & 0.0108 & 0.0520 & 0.0250 & 0.0216 & 0.0213 & 0.0447 & 0.0054 & 0.0054 & 0.0375 & 0.0416 & 0.0085 & 0.0115 & 0.0700 \\
\hline 0.0127 & 0.0108 & 0.0371 & 0.0250 & 0.0278 & 0.0244 & 0.0447 & 0.0054 & 0.0054 & 0.0125 & 0.0138 & 0.0142 & 0.0077 & 0.0077 \\
\hline
\end{tabular}

d. Determines the ideal positive matrix and the ideal negative matrix

The ideal solution is positive and negative; the ideal positive and negative solution can be determined based on the normalized weight rating. Please note the conditions on the positive and negative equations to calculate the value of the ideal solution by first determining whether benefit or cost.

1) Positive Solutions

$$
\begin{aligned}
& \text { Y1 + }=\operatorname{MAX}\{0,1149 ; 0.0127 ; 0.1149 ; 0.1149 ; 0.0127\}=0.1149 \\
& \text { Y2 + = MAX }\{0,0976 ; 0.0108 ; 0.0108 ; 0.0108 ; 0.0108\}=0.0976 \\
& \text { Y3-= MIN }\{0,0668 ; 0.0074 ; 0.0371 ; 0.0520 ; 0.0371\}=0.0074 \\
& \text { Y4-= MIN }\{0,0282 ; 0.0094 ; 0.0188 ; 0.0250 ; 0.0250\}=0.0094 \\
& \text { Y5-= MIN }\{0,0154 ; 0.0278 ; 0.0154 ; 0.0216 ; 0.0278\}=0.0154 \\
& \text { Y6-= MIN }\{0,0213 ; 0.0274 ; 0.0152 ; 0.0213 ; 0.0244\}=0.0152
\end{aligned}
$$


Abdul Azis, Bagus Adhi Kusuma, Alfika Maselia / IJIIS Vol. 3, No. 1, March 2020, pp. 1-11

Y7-= MIN $\{0,0447 ; 0.0447 ; 0.0447 ; 0.0447 ; 0.0447\}=0.0447$

$\mathrm{Y} 8+=\operatorname{MAX}\{0,0054 ; 0.0054 ; 0.0488 ; 0.0054 ; 0.0054\}=0.0488$

Y9-= MIN $\{0,0054 ; 0.0488 ; 0.0054 ; 0.0054 ; 0.0054\}=0.0054$

Y10- $=\operatorname{MIN}\{0,0125 ; 0.0250 ; 0.0125 ; 0.0375 ; 0.0125\}=0.0125$

Y11-= $\operatorname{MIN}\{0,0138 ; 0.0138 ; 0.0138 ; 0.0416 ; 0.0138\}=0.0138$

$\mathrm{Y} 12+=\operatorname{MAX}\{0,0142 ; 0.0085 ; 0.0085 ; 0.0085 ; 0.0142\}=0.0142$

Y13- $=\operatorname{MIN}\{0,0077 ; 0.0115 ; 0.0154 ; 0.0115 ; 0.0077\}=0.0077$

$\mathrm{Y} 14+=\operatorname{MAX}\{0,0700 ; 0.0077 ; 0.0077 ; 0.0700 ; 0.0077\}=0.0700$

2) Negative Solution

Y1-= MIN $\{0,1149 ; 0.0127 ; 0.1149 ; 0.1149 ; 0.0127\}=0.0127$

$\mathrm{Y} 2-=\operatorname{MIN}\{0,0976 ; 0.0108 ; 0.0108 ; 0.0108 ; 0.0108\}=0.0108$

$\mathrm{Y} 3+=\operatorname{MAX}\{0,0668 ; 0.0074 ; 0.0371 ; 0.0520 ; 0.0371\}=0.0668$

$\mathrm{Y} 4+=\operatorname{MAX}\{0,0282 ; 0.0094 ; 0.0188 ; 0.0250 ; 0.0250\}=0.0282$

$\mathrm{Y} 5+=\operatorname{MAX}\{0,0154 ; 0.0278 ; 0.0154 ; 0.0216 ; 0.0278\}=0.0278$

$\mathrm{Y} 6+=\operatorname{MAX}\{0,0213 ; 0.0274 ; 0.0152 ; 0.0213 ; 0.0244\}=0.0274$

$\mathrm{Y} 7+=\operatorname{MAX}\{0,0447 ; 0.0447 ; 0.0447 ; 0.0447 ; 0.0447\}=0.0447$

Y8-= MIN $\{0,0054 ; 0.0054 ; 0.0488 ; 0.0054 ; 0.0054\}=0.0054$

$\mathrm{Y} 9+=\operatorname{MAX}\{0,0054 ; 0.0488 ; 0.0054 ; 0.0054 ; 0.0054\}=0.0488$

$\mathrm{Y} 10+=\operatorname{MAX}\{0,0125 ; 0.0250 ; 0.0125 ; 0.0375 ; 0.0125\}=0.0375$

$\mathrm{Y} 11+=\operatorname{MAX}\{0,0138 ; 0.0138 ; 0.0138 ; 0.0416 ; 0.0138\}=0.0416$

Y12-= MIN $\{0,0142 ; 0.0085 ; 0.0085 ; 0.0085 ; 0.0142\}=0.0085$

$\mathrm{Y} 13+=\operatorname{MAX}\{0,0077 ; 0.0115 ; 0.0154 ; 0.0115 ; 0.0077\}=0.0154$

Y14-= MIN $\{0,0700 ; 0.0077 ; 0.0077 ; 0.0700 ; 0.0077\}=0.0077$

e. Specifies the distance between the values of each alternative with a matrix of positive and negative ideal solutions.

$$
D_{i}^{-}=\sqrt{\sum_{j=1}^{n}\left(y_{i j}-y_{j}^{-}\right)^{2}}
$$

Description:

$D_{i}^{+}:$alternative distance to-I of the ideal positive solution

$D_{i}^{-}:$alternative distance to-I of the ideal negative solution

1) Positive Solutions

$$
\mathrm{D} 1^{+}=\sqrt{\begin{array}{c}
(0.1149-0,1149)^{2}+(0,0976-0,0976)^{2}+(0,0074-0.0668)^{2}+ \\
(0.0094-0.0282)^{2}+(0,0154-0,0154)^{2}+(0,0152-0.0213)^{2} \\
+(0,0447-0,0447)^{2}+(0,0488-0,0054)^{2}+(0,0054-0,0054)^{2} \\
+(0.0125-0.0125)^{2}+(0,0138-0,0138)^{2}+(0,0142-0,0142)^{2} \\
+(0,0077-0,0077)^{2}+(0,0700-0,0700)^{2} \\
=0.0762
\end{array}}
$$




$$
\begin{aligned}
& (0,1149-0.0127)^{2}+(0,0976-0.0108)^{2}+(0,0074-0.0074)^{2}+ \\
& (0.0094-0.0094)^{2}+(0,0154-0,0278)^{2}+(0,0152-0,0274)^{2} \\
& \mathrm{D} 2^{+}=+(0,0447-0,0447)^{2}+(0,0488-0,0054)^{2}+(0,0054-0,0488)^{2} \\
& +(0.0125-0.0250)^{2}+(0,0416-0,0138)^{2}+(0,0142-0,0085)^{2} \\
& +(0,0154-0,0115)^{2}+(0,0700-0,0077)^{2} \\
& =0.1616 \\
& \mathrm{D}^{+}=\sqrt{\begin{array}{c}
(0,1149-0,1149)^{2}+(0,0976-0,0108)^{2}+(0,0074-0,0371)^{2}+ \\
(0,0282-0,0188)^{2}+(0,0154-0,0154)^{2}+(0,0244-0,0152)^{2} \\
+(0,0447-0,0447)^{2}+(0,0488-0,0488)^{2}+(0,0054-0,0054)^{2} \\
+(0,0125-0,0075)^{2}+(0,0416-0,0138)^{2}+(0,0142-0,0085)^{2} \\
+(0,0154-0,0154)^{2}+(0,0700-0,0077)^{2} \\
=0.1117
\end{array}} \sqrt{\sqrt{(0,1149-0,1149)^{2}+(0,0976-0,0108)^{2}+(0,0074-0,0520)^{2}+}} \\
& \mathrm{D} 4^{+}=\mid \begin{array}{c}
(0,0282-0,0250)^{2}+(0,0154-0,0216)^{2}+(0,0244-0,0213)^{2} \\
+(0,0447-0,0447)^{2}+(0,0488-0,0054)^{2}+(0,0054-0,0054)^{2} \\
+(0,0125-0,0375)^{2}+(0,0416-0,0416)^{2}+(0,0142-0,0085)^{2}
\end{array} \\
& +(0,0154-0,0115)^{2}+(0,0700-0,0700)^{2} \\
& =0.1147 \\
& \mathrm{D}^{+}=\sqrt{\begin{array}{c}
(0,1149-0,0127)^{2}+(0,0976-0,0108)^{2}+(0,0074-0,0371)^{2}+ \\
(0,0282-0,0250)^{2}+(0,0154-0,0278)^{2}+(0,0244-0,0244)^{2} \\
+(0,0447-0,0447)^{2}+(0,0488-0,0054)^{2}+(0,0054-0,0054)^{2} \\
+(0,0125-0,0125)^{2}+(0,0416-0,0138)^{2}+(0,0142-0,0142)^{2} \\
+(0,0154-0,0077)^{2}+(0,0700-0,0077)^{2} \\
=0.1585
\end{array}}
\end{aligned}
$$

2) Negative Solution

$$
\begin{aligned}
& (0,1149-0,0127)^{2}+(0,0976-0,0108)^{2}+(0,0668-0,0668)^{2} \\
& +\left(0,0282-0,0094+(0,0154-0,0278)^{2}+(0,0213-0,0152)^{2}\right. \\
& \mathrm{D} 1^{-}=+(0,0447-0,0447)^{2}+(0,0054-0,0054)^{2}+(0,0054-0,0488)^{2} \\
& +(0,0125-0,0375)^{2}+(0,0138-0,0138)^{2}+(0,0142-0,0085)^{2} \\
& \sqrt{+(0,0077-0,0077)^{2}+(0,0700-0,0077)^{2}}
\end{aligned}
$$$$
\text { D2 }=\sqrt{\begin{array}{c}
(0,0127-0,0127)^{2}+(0,0108-0,0108)^{2}+(0,0074-0,0668)^{2} \\
+(0,00984-0,0094)^{2}+(0,0278-0,0278)^{2}+(0,0274-0,0152)^{2} \\
+(0,0447-0,0447)^{2}+(0,0054-0,0054)^{2}+(0,0488-0,0488)^{2} \\
+(0,0250-0,0375)^{2}+(0,0138-0,0138)^{2}+(0,0085-0,0085)^{2} \\
+(0,0115-0,0077)^{2}+(0,0077-0,0077)^{2} \\
=0.0620
\end{array}}
$$

$(0,1149-0,0127)^{2}+(0,0108-0,0108)^{2}+(0,0371-0,0668)^{2}$

$\mathrm{D}^{-}=\sqrt[\begin{array}{c}+(0,0188-0,0094)^{2}+(0,0154-0,0278)^{2}+(0,0152-0,0152)^{2} \\ +(0,0447-0,0447)^{2}+(0,0488-0,0054)^{2}+(0,0054-0,0488)^{2} \\ +(0,0125-0,0375)^{2}+(0,0138-0,0138)^{2}+(0,0085-0,0085)^{2} \\ +(0,0154-0,0077)^{2}+(0,0077-0,0077)^{2}\end{array}=0.1265]{=}$

$$
\text { D4 }=\sqrt{\begin{array}{c}
(0,1149-0,0127)^{2}+(0,0108-0,0108)^{2}+(0,0520-0,0668)^{2} \\
+(0,0250-0,0094)^{2}+(0,0216-0,0278)^{2}+(0,0213-0,0152)^{2} \\
+(0,0447-0,0447)^{2}+(0,0054-0,0054)^{2}+(0,0054-0,0488)^{2} \\
+(0,0375-0,0375)^{2}+(0,0416-0,0138)^{2}+(0,0085-0,0085)^{2} \\
+(0,0115-0,0077)^{2}+(0,0700-0,0077)^{2} \\
=0.1324
\end{array}}
$$


Abdu1 Azis, Bagus Adhi Kusuma, Alfika Maselia / IJIIS Vol. 3, No. 1, March 2020, pp. 1-11

$$
\mathrm{D}^{-}=\sqrt{\begin{array}{c}
(0,0127-0,0127)^{2}+(0,0108-0,0108)^{2}+(0,0371-0,0668)^{2} \\
+\left(0,0250-0,0094+(0,0278-0,0278)^{2}+(0,0244-0,0152)^{2}\right. \\
+(0,0447-0,0447)^{2}+(0,0054-0,0054)^{2}+(0,0054-0,0488)^{2} \\
+(0,0125-0,0375)^{2}+(0,0138-0,0138)^{2}+(0,0142-0,0085)^{2} \\
+(0,0077-0,0077)^{2}+(0,0077-0,0077)^{2} \\
=0.0612
\end{array}}
$$

f. Specifying preference values for each alternative

$$
\begin{array}{lr}
V_{i}=\frac{D_{i}^{-}}{D_{i}^{-}+D_{i}^{+}} \\
V_{1}=\frac{0.1579}{0.1579+0.0792}=0,6658 & \text { Rank 1 =V1 } \\
V_{2}=\frac{0.0620}{0.0620+0.1646}=0,2736 & \text { Rank 2 =V4 } \\
V_{3}=\frac{0.1265}{0.1265+0.1154}=0,5228 & \text { Rank 3 =V3 } \\
V_{4}=\frac{0.1324}{0.1324+0.1102}=0,5457 & \text { Rank 4 =V5 } \\
V_{5}=\frac{0.0612}{0.0612+0.1600}=0,2766 & \text { Rank 5 =V2 }
\end{array}
$$

\section{Conclusions and Suggestions}

\subsection{Conclusions}

Based on the discussion and description in the previous chapters, it can be concluded as follows:

1) Based on the results of the study that the concentration support system for the determination of Low-Income Students Scholarship using the Technique for Order Preference by Similarity to Ideal Solution (TOPSIS) in Muhammadiyah 3 Middle School in Purwokerto has been successfully created. The final result of the system shows fourteen criteria that have different weights in each criterion, as well as generating a rank of the highest value to the lowest gained from the calculation decision making Low-Income Students Scholarship.

2) With a web system that has successfully created student data and calculation of Low-Income Students Scholarship stored securely in the system database and can be accessed easily.

\subsection{Suggestions}

Based on the conclusion of the results of this study, it can be suggested for subsequent studies are as follows:

1) In the next research, it would be better if the current system for the future is developed again to be based on Android.

2) The decision-making system for Low-Income Students Scholarship will be developed by other decision-making methods so that they show similarities or differences in results.

\section{References}

[1] Melnyk, S. A., Sroufe, R. P., \& Calantone, R. (2003). Assessing the impact of environmental management systems on corporate and environmental performance. Journal of Operations Management, 21(3), 329-351.

[2] Messeni Petruzzelli, A., Maria Dangelico, R., Rotolo, D., \& Albino, V. (2011). Organizational factors and technological features in the development of green innovations: Evidence from patent analysis. Innovation, 13(3), 291-310.

[3] Min, H., \& Galle, W. P. (2001). Green purchasing practices of US firms.International Journal of Operations \& Production Management, 21(9), 1222-1238.

[4] Montalvo, C. (2003). Sustainable production and consumption systems - cooperation for change: assessing and simulating the willingness of the firm to adopt/develop cleaner technologies. The case of the In-Bond industry in northern Mexico. Journal of cleaner production, 11(4), 411-426. 12 
Abdu1 Azis, Bagus Adhi Kusuma, Alfika Maselia / IJIIS Vol. 3, No. 1, March 2020, pp. 1-11

[5] Nelson, R. R., \& Winter, S. G. (2009). An evolutionary theory of economic change. Harvard University Press.

[6] Nikbakhsh, E. (2009). Green supply chain management. In Supply chain and logistics in national, international and governmental environment (pp. 195-220). Physica-Verlag HD.

[7] Noci, G., \& Verganti, R. (1999). Managing 'green'product innovation in small firms. R\&d Management, 29(1), 3-15.

[8] Oltra, V., \& Saint Jean, M. (2009). Sectoral systems of environmental innovation: an application to the French automotive industry. Technological Forecasting and Social Change, 76(4), 567- 583.

[9] Olugu, E. U., Wong, K. Y., \& Shaharoun, A. M. (2011). Development of key performance measures for the automobile green supply chain. Resources, conservation and recycling, 55(6), 567-579. 